Objectives: This study evaluated the efficacy and safety of FEN compared with placebo (PBO) and adalimumab (ADA), in combination with background methotrexate (MTX), in patients (pts) with rheumatoid arthritis (RA)

Methods: This multicenter, randomized, double-blind Phase 2 study included pts with moderate-to-severe active RA with an inadequate response to MTX (MTXIR, Cohort 1) or TNF inhibitors (TNF-IR, Cohort 2). Cohort 1 pts were randomized to FEN at $50 \mathrm{mg}$ QD (FEN-50), $150 \mathrm{mg}$ QD (FEN-150), $200 \mathrm{mg}$ BID (FEN-200), 40 mg ADA injections SC Q2W, or PBO. Cohort 2 pts were randomized to FEN-200 or PBO. Key efficacy endpoints evaluated the proportion of pts with an ACR50 response at Week 12 (W12), comparing FEN doses to PBO (both cohorts) and to ADA (Cohort 1).

Results: Cohort 1 (FEN-50, $n=40$; FEN-150, $n=109$; FEN-200, $n=110$; PBO, $n=110 ; A D A, n=111)$ and Cohort $2(F E N-200, n=48 ; P B O, n=50)$ demographics and disease characteristics were balanced, and $\sim 90 \%$ of pts per arm completed the study. In Cohort 1, ACR50 response rates increased with increasing FEN dose $(18 \%, 28 \%$, and $35 \%$ for FEN-50, FEN-150, and FEN-200, respectively). FEN-150 (28\%, p=0.0164) and FEN-200 (35\%, p=0.0003) were superior to PBO (15\%), and numerically similar to ADA (36\%). In Cohort 2, the response for FEN200 was higher than PBO (25\% vs. $12 \%$ ) (Table 1). Adverse events (AEs) were generally balanced across Cohort 1; there were 9 serious AEs in 7 pts and one death in the FEN-200 group. In Cohort 2, more pts in the PBO arm reported AEs, and no serious AEs were reported.

Conclusion: FEN demonstrated higher efficacy rates than PBO for ACR50 at W12 in both MTX-IR and TNF-IR populations, and was similar to ADA in MTX-IR pts. The overall safety profile of FEN was acceptable.

Disclosure of Interests: Stanley Cohen Grant/research support from: AbbVie, Amgen Inc., AstraZeneca, Biogen-IDEC, Bristol Meyer Squibb, Genentech, Janssen, Eli Lilly, Novartis, Pfizer, Merck, and Roche, Consultant for: Abbvie, Amgen, AstraZeneca, Biogen-IDEC, Bristol Meyer Squibb, Genentech, Janssen, Lilly, Novartis Pfizer, Merck and Roche, Katie Tuckwell Shareholder of: Genentech/ Roche, Employee of: Genentech/Roche, Tamiko R. Katsumoto Shareholder of: Genentech, Inc., Consultant for: Roche/Genentech, Principia Biopharma, Abbvie, Employee of: Former employee of Genentech, Inc., Rui Zhao Shareholder of: Genentech, Inc., Employee of: Genentech, Inc., Chin Lee Shareholder of: Roche/ Genentech and of Eli Lilly \& Co., Employee of: Genetech, Inc. and Eli Lilly \& Co. Alberto Berman Grant/research support from: Grants/research support: Roche/ Genentech, Bristol-Myers Squibb, Merck Serono, AbbVie, Amgen, Eli Lilly, Janssen, Nemanja Damjanov Grant/research support from: AbbVie, Pfizer and Roche, Consultant for: Abbvie, Gedeon Richter, Merck, Novartis, Pfizer and Roche., Speakers bureau: Abbvie, Gedeon Richter, Merck, Novartis, Pfizer and Roche., Dmytro Fedkov Grant/research support from: MSD, AbbVie, ProPharma, Laboratoires Expanscience; Consultant for: MSD, AbbVie, ProPharma, Laboratoires Expanscience; Speakers bureau: Janssen, Sławomir Jeka: None declared, Mark C. Genovese Grant/research support from: Sanofi/Genzyme, Genentech/Roche, RPharm, Consultant for: Sanofi/Genzyme, Genentech/Roche, RPharm DOI: 10.1136/annrheumdis-2019-eular.4469

\section{OP0026 A PHASE 3 STUDY OF THE EFFICACY AND SAFETY OF PEFICITINIB (ASP015K) IN PATIENTS WITH RHEUMATOID ARTHRITIS WHO HAD AN INADEQUATE RESPONSE TO METHOTREXATE}

Tsutomu Takeuchi ${ }^{1}$, Yoshiya Tanaka ${ }^{2}$, Sakae Tanaka ${ }^{3}$, Atsushi Kawakami ${ }^{4}$ Manabu Iwasaki ${ }^{5}$, Mitsuhiro Rokuda ${ }^{6}$, Hiroyuki Izutsu ${ }^{6}$, Satoshi Ushijima ${ }^{6}$, Yuichiro Kaneko 6 , Teruaki Shiomi ${ }^{6}$, Emi Yamada ${ }^{6} .{ }^{1}$ Keio University, Tokyo, Japan; ${ }^{2}$ University of Occupational and Environmental Health, Kitakyushu, Japan; ${ }^{3}$ University of Tokyo, Tokyo, Japan; ${ }^{4}$ Nagasaki University, Nagasaki, Japan; ${ }^{5}$ Yokohama City University, Yokohama, Japan; ${ }^{6}$ Astellas Pharma, Inc., Tokyo, Japan

Background: Peficitinib (ASP015K), a novel oral JAK inhibitor, demonstrated efficacy as once-daily monotherapy in patients with moderate-to-severe rheumatoid arthritis (RA) in a phase $2 \mathrm{~b}$ study (NCT01649999) ${ }^{1}$.

Objectives: To evaluate the efficacy and safety of peficitinib-methotrexate (MTX) combination in patients with RA who had an inadequate response to MTX. Methods: This multicentre, randomised, double-blind, parallel-group, placebo (PBO)-controlled, phase 3 study (NCT02305849) was conducted in Japan. Patients had RA diagnosed within the past 10 years (1987 ACR or 2010 ACR/ EULAR criteria), active disease ( $\geq 6$ tender and painful joints and $\geq 6$ swollen joints, using 68 and 66 -joint assessment respectively; CRP $\geq 1.0 \mathrm{mg} / \mathrm{dL}$; bone erosion; and ACPA or RF positivity) and inadequate response to MTX (administered for $\geq 90$ days; $\geq 8 \mathrm{mg} /$ week for $\geq 28$ days prior to baseline). Patients were randomised 1:1:1 to 52-week MTX plus PBO, peficitinib $100 \mathrm{mg} /$ day or peficitinib 150 $\mathrm{mg} /$ day. At week 12, inadequate responders in the PBO group ( $<20 \%$ improvement from baseline in tender and swollen joint counts) were switched (under blinded conditions) to peficitinib 100/150 mg until end of treatment. Remaining patients in the PBO group were switched (under blinded conditions) to peficitinib at week 28. Concomitant stable MTX dose ( $\leq 16 \mathrm{mg} /$ week) was mandatory.
Primary efficacy variables were ACR20 response rate at week 12/early termination (ET) and change from baseline in modified Total Sharp score (mTSS) at week 28/ET.

Table 1 Primary and selected secondary efficacy endpoints at week 12/ET

\begin{tabular}{|c|c|c|c|c|c|c|}
\hline & \multicolumn{3}{|c|}{ Week 12/ET } & \multicolumn{3}{|c|}{ Week 28/ET } \\
\hline Result & PBO & \begin{tabular}{|l|} 
Peficitinib \\
$100 \mathrm{mg} / \mathrm{day}$
\end{tabular} & $\begin{array}{l}\text { Peficitinin } \\
150 \mathrm{mg} / \mathrm{day}\end{array}$ & PBO & \begin{tabular}{|l|}
$\begin{array}{l}\text { Peficitininib } \\
100 \mathrm{mg} / \text { day }\end{array}$ \\
\end{tabular} & \begin{tabular}{|l|} 
Peficititinib \\
$150 \mathrm{mg} /$ day
\end{tabular} \\
\hline$\overline{A C R 20, n / N(\%)}$ & \begin{tabular}{|l|}
$37 / 170(21.8)$ \\
\end{tabular} & \begin{tabular}{|l|}
$102 / 174$ \\
$558.6 \%{ }^{* * *}$
\end{tabular} & $\begin{array}{l}112 / 1744 \\
\left\{64.44^{* * *}\right.\end{array}$ & $50 / 170(29.4)$ & $\begin{array}{l}129 / 174 \\
174.11^{* * * *}\end{array}$ & \begin{tabular}{|l|}
$137 / 174$ \\
$178.77^{* * *}$
\end{tabular} \\
\hline$\overline{A C R 50, n / N(\%)}$ & $13 / 170(7.6)$ & $52 / 174(29.9)^{* * *}$ & $80 / 174(46.0)^{* * *}$ & $\begin{array}{l}19 / 170\{11.2\} \\
\end{array}$ & $88 / 174(50.6)^{* * *}$ & \begin{tabular}{l|}
$103 / 174$ \\
$(159.2)^{2 * \times x}$
\end{tabular} \\
\hline$A C R 70, n / N(\%)$ & $4 / 170(2.4)$ & $21 / 174(12.1)^{* * *}$ & $41 / 174(23.6)^{* * *}$ & $10 / 170\{5.9)$ & $47 / 174(27.0)^{* * *}$ & $70 / 174(40.2)^{* *}$ \\
\hline $\begin{array}{l}\text { Mean (SD) CRP } \\
\text { change from } \\
\text { baseline } \mathrm{mg} / \mathrm{dL}\end{array}$ & \begin{tabular}{|l|}
$-0.001(2.038)$ \\
\end{tabular} & $-1.499(1.855)^{* * *}$ & $-1.421(2.182)^{* * * x}$ & \begin{tabular}{|l|}
$0.041(2.399)$ \\
\end{tabular} & $-1.649(2.165)^{* x *}$ & $-1.625(2.236)^{* * *}$ \\
\hline $\begin{array}{l}\text { Mean (SD) ESR } \\
\text { change from } \\
\text { baseline, } \mathrm{mm} / \mathrm{h}\end{array}$ & \begin{tabular}{|l|}
$-2.42(19.71)$ \\
\end{tabular} & $-18.90\{19.85\}^{* * 4}$ & $-22.17(22.79)^{* * 4}$ & \begin{tabular}{|l|}
$-3.26(22.68)$ \\
\end{tabular} & $-24.51(23.67)^{* * *}$ & \begin{tabular}{|l|}
$-26.21(24.58)^{* * *}$ \\
\end{tabular} \\
\hline $\begin{array}{l}\text { DAS28-CRP < <2.6, } \\
\mathrm{N} / \mathrm{N}(\%)\end{array}$ & $13 / 169\{(7.7)$ & $54 / 172(31.9)^{* * *}$ & $60 / 171(35.1)^{* * * *}$ & $200 / 169\{11.8\}$ & $86 / 172(50.0)^{* * * *}$ & $86 / 171(50.3)^{* * *}$ \\
\hline $\begin{array}{l}\text { Mean (SD) DAS28- } \\
\text { CRP change from } \\
\text { baseline }\end{array}$ & $-0.51(1.10)$ & $-1.70\{1.20\}^{* * *}$ & $-2.09(1.33)^{* * *}$ & $-0.64\{1.33\}$ & $-2.27(1.31)^{* * *}$ & $-2.56(1.38)^{* * *}$ \\
\hline $\begin{array}{l}\text { Mean (SD) change } \\
\text { from baseline in } \\
\text { patient's } \\
\text { assessment of } \\
\text { gain, } 100 \text { mm VAS }\end{array}$ & $\begin{array}{l}-6.64(25.22) \\
\end{array}$ & $-21.09\{27.04)^{* * 2}$ & $-26.87(26.65)^{* * *}$ & $-7.61(27.81)$ & $-26.33(28.22)^{* * *}$ & $-32.23(27.59)^{k * *}$ \\
\hline \multirow[t]{2}{*}{$\begin{array}{l}\text { SDAl remission } \\
\text { (SDAl score } \leq 3.3), \\
n / N(\%)\end{array}$} & $1 / 169\{0.6)$ & $12 / 172(7.0)^{* *}$ & $24 / 171(14.0)^{* * *}$ & $6 / 169\{3.6\}$ & $36 / 172(20.9)^{* * *}$ & $37 / 171(21.6)^{* * *}$ \\
\hline & PBO & $\begin{array}{l}\text { Week 28/ET } \\
\text { Peficitinib } \\
100 \mathrm{me} / \text { day }\end{array}$ & $\begin{array}{l}\text { Peficitinin } \\
150 \mathrm{~m} / \text { day }\end{array}$ & PBO & $\begin{array}{l}\text { Week 52/ET } \\
\begin{array}{l}\text { Peficitinib } \\
100 \mathrm{mg} / \text { day }\end{array} \\
\end{array}$ & \begin{tabular}{|l|}
$\begin{array}{l}\text { Peficitininib } \\
150 \mathrm{mg} / \text { day }\end{array}$ \\
\end{tabular} \\
\hline $\begin{array}{l}\text { Mean (SD) mTSS } \\
\text { change from } \\
\text { baseline? }\end{array}$ & $3.37(5.46)$ & $1.62(4.23)^{* * *}$ & $1.03(2.86)^{* * *}$ & $6.27(10.18)$ & $2.12(5.83)^{* * *}$ & $1.54(4.11)^{* * *}$ \\
\hline $\begin{array}{l}\text { Patients achieving } \\
\text { mean mTSS } \\
\text { change from } \\
\text { baseline so.5\%, } \\
\mathrm{n} / \mathrm{N}(\%)\end{array}$ & $70 / 153(45.8)$ & $\begin{array}{l}110 / 164 \\
\langle 67.1)^{* * x}\end{array}$ & $\begin{array}{l}119 / 164 \\
(72.6)^{\mathrm{k**}}\end{array}$ & $65 / 153(42.5)$ & $\begin{array}{l}105 / 164 \\
\{64.0)^{* \times \times}\end{array}$ & \begin{tabular}{|l|l|}
$113 / 164$ \\
$\langle 68.9\rangle^{* \times x}$
\end{tabular} \\
\hline
\end{tabular}

Last Observation Carried Forward imputation method

peficitinib $100 \mathrm{mg}$, and $150 \mathrm{mg}$, compared with PBO.
$*$ "p 00.01 vs PBO; *** $\mathrm{p}<0.001$ vs PBO, according to Fisher's Exact test for ACR20, ACR50, ACR70, DAS28-CRP $<2.6,5 D A$ remission, and mTSS change from baseline $\leq 0.5$; analysis of covariance for CRP, ESR, DAS28-CRP, and patient's assessment of pain; and rank analysis of covariance
adjustment in the primary analysis.

"For the calculation of mTSS, patients who discontinued at or before week 28 or were switched from $\mathrm{PBO}$ to peficitinib week 12 due to lack of efficacy, week 28/ET mTSS was extrapolated using linear extrapolation method based on the mTSS at baseline and early termination or week 12 (day 85) (before switching). For patients who discontinued at or before week 52 or switched to receive peficitinib instead of placebo at week 12 or week 28 , $\mathrm{mTSS}$ at week $52 / \mathrm{ET}$ was extrapolated using a line extrapolation

Switching).
mTSS=modified Total Sharp score; SD=standard deviation; SDAl=simplified disease activity index; VAS=visual analog scale.

Table 2 Treatment-emergent adverse events

\begin{tabular}{|c|c|c|c|c|}
\hline & $P B O\{N=170)$ & \begin{tabular}{|l|} 
Peficitinib \\
$100 \mathrm{mg} /$ day \\
(N=174)
\end{tabular} & \begin{tabular}{|l|} 
Peficitinib \\
$150 \mathrm{mg} /$ day \\
(N=174)
\end{tabular} & \begin{tabular}{|l|} 
Peficitinib \\
$100 \mathrm{mg} /$ day + \\
$150 \mathrm{mg} /$ day \\
$(\mathrm{N}=348)$ \\
\end{tabular} \\
\hline \multicolumn{5}{|l|}{ eek 0-12 } \\
\hline Any $\mathrm{AE}$ & $84(49.4)$ & $89(51.1)$ & $104(59.8)$ & $193(55.5)$ \\
\hline Drug-related $\mathrm{AE}^{\mathrm{a}}$ & $47(27.6)$ & $57(32.8)$ & $80(46.0)$ & $137(39.4)$ \\
\hline Death & 0 & 0 & 0 & 0 \\
\hline SAE & $4(2.4)$ & $5(2.9)$ & $3(1.7)$ & $8(2.3)$ \\
\hline Drug-related SAE & $2(1.2)$ & $3(1.7)$ & $3(1.7)$ & $6(1.7)$ \\
\hline Grade $\angle 3 \mathrm{AE}^{\mathrm{b}}$ & $8(4.7)$ & $9(5.2)$ & $16(9.2)$ & $25(7.2)$ \\
\hline $\begin{array}{l}\text { AE leading to permanent } \\
\text { discontinuation of study drug }\end{array}$ & $7(4.1)$ & $5(2.9)$ & $5(2.9)$ & $10(2.9)$ \\
\hline Serious infection & 0 & $3(1.7)$ & $1(0.6)$ & $4(1.1)$ \\
\hline Herpes zoster-related disease & 0 & $2(1.1)$ & $3(1.7)$ & $5(1.4)$ \\
\hline Malignancy & 0 & 0 & 0 & 0 \\
\hline \begin{tabular}{|l|} 
Incidence rate per 100 patient- \\
years ( $95 \%$ confidence interval), \\
overall study periodc
\end{tabular} & $\mathrm{PBO}\{\mathrm{N}=170\}$ & \begin{tabular}{|l} 
Peficitinib \\
$100 \mathrm{mg} /$ day \\
(N=174)
\end{tabular} & \begin{tabular}{|l} 
Peficitinib \\
$150 \mathrm{mg} /$ day \\
$(\mathrm{N}=174)$
\end{tabular} & $\begin{array}{l}\text { Peficitinib total } \\
(\mathrm{N}=496)^{\circ}\end{array}$ \\
\hline Serious infectione & 0.0 & $3.8(1.7,8.4)$ & $3.7(1.7,8.3)$ & $3.4(2.0,5.8)$ \\
\hline Herpes zoster-related disease & $3.2(0.8,12.8)$ & $8.3(4.8,14.3)$ & $3.8(1.7,8.4)$ & $5.7(3.8,8.6)$ \\
\hline Malignancy & $1.6(0.2,11.3)$ & $0.6(0.1,4.4)$ & 0.0 & $0.2(0.0,1.7)$ \\
\hline
\end{tabular}

ossibly or probably related to study drug, as assessed by the investigator, or records where relationship is missing. Based on NCI-CTCAE rade: grade 3 -severe or medically significast, cPatient-years covers from initial dose up to first incidence of AE for patients who had at least one event, otherwise, the duration of the patients are summed as from initial dose through follow-up. Incidence rate was calculated as (100 x number of patients who had at least one incidence / total patient-yearl.

Heforicitin $100 \mathrm{mg} /$ day or $150 \mathrm{mg} / \mathrm{day}$

DE=adverse event: SAE=serious adverse event

Results: 519 patients were treated: PBO $(n=170)$, peficitinib $100 \mathrm{mg}(n=175)$ and peficitinib $150 \mathrm{mg}(\mathrm{n}=174)$. At week 12, 75 PBO-treated patients were switched to peficitinib $100 \mathrm{mg}(\mathrm{n}=37)$ and $150 \mathrm{mg}(\mathrm{n}=38)$ due to inadequate response. At week 12/ET, peficitinib showed superior efficacy vs PBO with respect to symptoms and inflammatory markers (Table 1). At weeks 28 and 52, peficitinib significantly reduced the mean mTSS change from baseline vs PBO (Table 1). Week 0-12 safety results were similar for $\mathrm{PBO}$ and peficitinib (Table 2). For the overall study period, incidence rate of serious infections per 100 patient-years was higher with peficitinib $100 \mathrm{mg} / 150 \mathrm{mg}$ than PBO (Table 2).

Conclusion: In patients with RA who had an inadequate response to MTX, peficitinib $100 \mathrm{mg} /$ day and $150 \mathrm{mg} /$ day demonstrated significant superiority vs PBO in reducing $R A$ symptoms and suppressing joint destruction, according to primary efficacy variables (ACR response and change in mTSS). Peficitinib $100 \mathrm{mg}$ and $150 \mathrm{mg}$ showed acceptable safety and tolerability, with no new safety signals compared with other JAK inhibitors. 
REFERENCE:

[1] Takeuchi T, et al. Ann Rheum Dis 2016; 75: 1057-64

Acknowledgement: This study was sponsored by Astellas Pharma, Inc. Medical writing support was provided by Rhian Harper Owen of Cello Health MedErgy and funded by Astellas Pharma, Inc.

Disclosure of Interests: Tsutomu Takeuchi Grant/research support from: Astellas Pharma Inc, Chugai Pharmaceutical Co, Ltd., Daiichi Sankyo Co., Ltd., Takeda Pharmaceutical Co., Ltd., AbbVie GK, Asahikasei Pharma Corp., Mitsubishi Tanabe Pharma Co., Pfizer Japan Inc., Eisai Co., Ltd., AYUMI Pharmaceutical Corporation, Nipponkayaku Co. Ltd., Novartis Pharma K.K., Grant/research support from: AbbVie, Asahi Kasei, Astellas, AstraZeneca, AYUMI, Bristol-Myers Squibb, Chugai, Daiichi Sankyo, Eisai, Eli Lilly Japan, Janssen, Mitsubishi Tanabe, Nippon Kayaku, Novartis, Pfizer Japan Inc, Taiho, Taisho Toyama, Takeda, Teijin, Grant/ research support from: Astellas Pharma Inc., Bristol Myers Squibb, Chugai Pharmaceutical Co., Ltd., Mitsubishi Tanabe Pharma Co., Pfizer Japan Inc., Santen Pharmaceutical Co., Ltd., Takeda Pharmaceutical Co., Ltd., Teijin Pharma Ltd., AbbVie GK, Asahi Kasei Pharma Corp., Taisho Toyama Pharmaceutical Co., Ltd. SymBio Pharmaceuticals Ltd., Janssen Pharmaceutical K.K., Celltrion Inc., Nipponkayaku Co. Ltd., and UCB Japan, Consultant for: Astra Zeneca K.K., Eli Lilly Japan K.K., Novartis Pharma K.K., Mitsubishi Tanabe Pharma Co., Abbivie GK, Nipponkayaku Co.Ltd, Janssen Pharmaceutical K.K., Astellas Pharma Inc., Taiho Pharmaceutical Co. Ltd., Chugai Pharmaceutical Co. Ltd., Taisho Toyama Pharmaceutical Co. Ltd., GlaxoSmithKline K.K., UCB Japan Co. Ltd., Consultant for: AbbVie, Asahi Kasei, Astellas, AstraZeneca, AYUMI, Bristol-Myers Squibb, Chugai, Daiichi Sankyo, Eisai, Eli Lilly Japan, Janssen, Mitsubishi Tanabe, Nippon Kayaku, Novartis, Pfizer Japan Inc, Taiho, Taisho Toyama, Takeda, Teijin, Consultant for: Astra Zeneca K.K., Eli Lilly Japan K.K., Novartis Pharma K.K., Mitsubishi Tanabe Pharma Co., Asahi Kasei Medical K.K., AbbVie GK, Daiichi Sankyo Co., Ltd., Bristol Myers Squibb, and Nipponkayaku Co. Ltd., Speakers bureau: Astellas Pharma Inc., Bristol Myers Squibb, Chugai Pharmaceutical Co., Ltd., Mitsubishi Tanabe Pharma Co., Pfizer Japan Inc., Santen Pharmaceutical Co., Ltd., Takeda Pharmaceutical Co., Ltd., Teijin Pharma Ltd., AbbVie GK, Asahi Kasei Pharma Corp., Taisho Toyama Pharmaceutical Co., Ltd., SymBio Pharmaceuticals Ltd., Janssen Pharmaceutical K.K., Celltrion Inc., Nipponkayaku Co. Ltd., and UCB Japan, Speakers bureau: AbbVie, Asahi Kasei, Astellas, AstraZeneca, AYUMI, Bristol-Myers Squibb, Chugai, Daiichi Sankyo, Eisai, Eli Lilly Japan, Janssen, Mitsubishi Tanabe, Nippon Kayaku, Novartis, Pfizer Japan Inc, Taiho, Taisho Toyama, Takeda, Teijin, Speakers bureau: AbbVie GK., Bristol-Myers K.K., Chugai Pharmaceutical Co. Ltd., Mitsubishi Tanabe Pharma Co., Pfizer Japan Inc., Astellas Pharma Inc, Diaichi Sankyo Co. Ltd., Eisai Co. Ltd., Sanofi K.K., Teijin Pharma Ltd., Takeda Pharmaceutical Co. Ltd., Novartis Pharma K.K., Yoshiya Tanaka Grant/research support from: Abbvie, Astellas, Bristol-Myers Squibb, Chugai, Daiichi-Sankyo, Eisai, Mitsubishi-Tanabe, MSD, Ono, TaishoToyama, Takeda, Speakers bureau: Abbvie, Asahi-kasei, Astellas, Bristol-Myers Squibb, Chugai, Daiichi-Sankyo, Eli Lilly, Eisai, Glaxo-Smithkline, Janssen, Mitsubishi-Tanabe, Novartis, Pfizer Japan Inc, Sanofi, Takeda, UCB, YL Biologics, Sakae Tanaka Grant/research support from: KYOCERA Corporation and Asahi Kasei Corporation, Consultant for: Amgen Astellas BioPharma K.K., KYOCERA Corporation, Pfizer and Daiichi Sankyo Co., Ltd., Speakers bureau: Asahi Kasei Corporation, Astellas Pharma Inc, Ayumi Pharmaceutical Corporation, Eisai Co., Ltd., Ono Pharmaceutical Co., Ltd., Daiichi Sankyo Co., Ltd, Taisho Toyama Pharmaceutical Co., Ltd., Mitsubishi Tanabe pharma Corporation, Chugai Pharmaceutical Co., Ltd., Teijin Pharma Ltd., Eli Lilly, Hisamitsu Pharmaceutical Co, Inc., Pfizer, Bristol-Myers., Atsushi Kawakami Grant/research support from: Astellas Pharma, Consultant for: Astellas Pharma, Speakers bureau: Astellas Pharma, Manabu Iwasaki: None declared, Mitsuhiro Rokuda Employee of: Astellas Pharma, Inc., Hiroyuki Izutsu Employee of: Astellas Pharma, Inc., Satoshi Ushijima Employee of: Astellas Pharma, Inc., Yuichiro Kaneko Employee of: Astellas Pharma, Inc., Teruaki Shiomi Employee of: Astellas Pharma, Inc., Emi Yamada Employee of: Astellas Pharma, Inc. DOI: 10.1136/annrheumdis-2019-eular.1580

\section{OP0027 \\ EFFECT OF SHORT-TERM METHOTREXATE DISCONTINUATION ON THE DISEASE ACTIVITY IN PATIENTS WITH RHEUMATOID ARTHRITIS: POSTHOC-ANALYSIS OF TWO RANDOMIZED CLINICAL TRIALS}

Min Jung Kim, Yeong Wook Song, Eun Bong Lee, Jin Kyun Park. Seoul National University Hospital, Seoul, Division of Rheumatology, Department of Internal Medicine, Seoul, Korea, Rep. of (South Korea)

Background: Patients with rheumatoid arthritis (RA) require a continuous, potentially life-long immune suppression with disease modifying antirheumatic drugs (DMARDs) including methotrexate (MTX). However, in special circumstances such as life-threatening infections, vaccinations or major surgeries, use of MTX should be minimized to restore the treatment-associated immune suppression. While a long-term or permanent discontinuation of MTX is associated with a disease flare or relapse, the effect of short-term discontinuation on disease activity has not been fully elucidated.

Objectives: To investigate the effect of short-term discontinuation of MTX on the disease activity in patients with RA on stable dose of MTX.

Methods: This is a posthoc analysis of 2 randomized controlled studies investigating effect of MTX discontinuation for 2 weeks or 4 weeks on vaccine response to seasonal influenza vaccination in patients with RA. In the 4-week discontinuation study, 54 patients continued MTX and 44 patients discontinued it for 4 weeks before vaccination with trivalent seasonal influenza vaccine. In the 2 -week discontinuation study, 159 patients continued MTX and 161 patients held it for 2 weeks after a seasonal quadrivalent influenza vaccine. Disease activity (DAS28 change, DAS28 flare rate and flare-free survival) was compared between the patients who continued MTX and those held it. A RA flare was defined as an increase in DAS28 of $>1.2$ or $>0.6$ if the baseline DAS28 was $\geq 3.2$.

Results: In the 4-week MTX-hold group, the mean DAS28 increased at the 4 weeks after MTX discontinuation by $0.38 \pm 0.94$ and then improved back to baseline after reintroduction of MTX, whereas the mean DAS28 in the MTX-continue group remained stable over time (Figure $1 \mathrm{~A}$ ). The overall flare-free survival during 20 weeks did not differ between the groups (log rank $p=0.142)$ (Figure 1B). However numerically more patients in the MTX-hold group experienced a flare than those in the MTX-continue group during the 4-weeks MTX discontinuation ( $20.5 \%$ vs. $7.4 \%$, $p=0.058$ ). After resuming MTX, the flare rate did not differ between the groups up to 20 weeks of observations (Figure $1 \mathrm{C}$ ). A temporary MTX discontinuation for 2 weeks was not associated with any clinically meaningful change in disease activity.

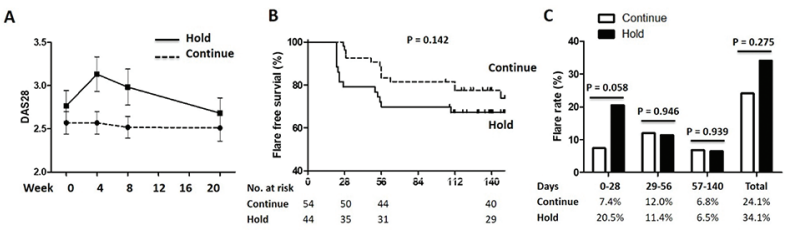

Figure 1. Changes in disease activity of rheumatoid arthritis after MTX discontinuation over time. (A) DAS28-CRP and (B) RA flare free survival in the MTX continue and the MTX hold group were depicted. (C) RA flare rates over time were shown. MTX, methotrexate.

Conclusion: A short-term MTX discontinuation for 2 weeks is safe without any change in disease activity. A 4-week MTX discontinuation is associated with transient increase in disease activity without affecting long-term outcomes.

\section{REFERENCES:}

[1] Park JK, Lee MA, Lee EY, Song YW, Choi Y, Winthrop KL, et al. Effect of methotrexate discontinuation on efficacy of seasonal influenza vaccination in patients with rheumatoid arthritis: a randomised clinical trial. Ann Rheum Dis. 2017 May 03.

[2] Park JK, Lee YJ, Shin K, Ha YJ, Lee EY, Song YW, et al. Impact of temporary methotrexate discontinuation for 2 weeks on immunogenicity of seasonal influenza vaccination in patients with rheumatoid arthritis: a randomised clinical trial. Ann Rheum Dis. 2018 Jun; 77(6):898-904.

[3] Haschka J, Englbrecht M, Hueber AJ, Manger B, Kleyer A, Reiser M, et al Relapse rates in patients with rheumatoid arthritis in stable remission tapering or stopping antirheumatic therapy: interim results from the prospective randomised controlled RETRO study. Ann Rheum Dis. 2016 Jan; 75(1):45-51.

Disclosure of Interests: None declared

DOI: 10.1136/annrheumdis-2019-eular.2524

\section{OP0028 POST-APPROVAL COMPARATIVE SAFETY STUDY OF TOFACITINIB AND BIOLOGIC DMARDS: FIVE-YEAR RESULTS FROM A US-BASED RHEUMATOID ARTHRITIS REGISTRY}

Joel Kremer ${ }^{1}$, Clifton Bingham ${ }^{2}$, Laura Cappelli ${ }^{2}$, Carol Etzel $^{3}$, Jeffrey Greenberg ${ }^{3}$, Jamie Geier ${ }^{4}$, Ann Madsen ${ }^{4}$, Connie Chen ${ }^{4}$, Alina Onofrei ${ }^{3}$, Christine Barr ${ }^{3}$, Dimitrios A. Pappas ${ }^{5}$, Heather J. Litman ${ }^{3}$, Kimberly J. Dandreo ${ }^{3}$, Andrea Shapiro ${ }^{6}$, Carol A. Connell ${ }^{7}$, Arthur Kavanaugh ${ }^{8}$. ${ }^{1}$ Albany Medical College, Center for Rheumatology, Albany, NY, United States of America; ${ }^{2}$ Johns Hopkins University, Baltimore, MD, United States of America; ${ }^{3}$ Corrona, LLC, Waltham, MA, United States of America; ${ }^{4}$ Pfizer Inc, New York, NY, United States of America; ${ }^{5}$ Colombia University, Department of Medicine, New York, NY, United States of America; ${ }^{6}$ Pfizer Inc, Peapack, NJ, United States of America; ${ }^{7}$ Pfizer Inc, Groton, CT, United States of America; ${ }^{8}$ University of California, San Diego School of Medicine, La Jolla, San Diego, CA, United States of America

Background: Tofacitinib is an oral JAK inhibitor for the treatment of rheumatoid arthritis (RA). Real-world data (RWD) complement clinical trial data in assessing 